\title{
Patient-reported outcome measures after COVID-19: a prospective cohort study
}

\author{
To the Editor:
}

Patient reported outcome measures (PROMs) are standardised validated questionnaires completed by patients to provide information on their perceived functional well-being and health status [1]. These questionnaires can address various aspects of health including symptoms, quality of life, functionality, and physical, mental and social well-being. PROMs play an important role in increasing patient engagement, improving health systems, and ensuring that clinical care and research is person-centred.

Initial research efforts in coronavirus disease 2019 (COVID-19) have appropriately been focused on its acute presentation and management. Over time, there have been studies investigating the short-term sequelae after COVID-19 with a focus on physiological and radiological abnormalities [2]. Studies exploring health status from the patient perspective have mainly evaluated the presence or absence of persistent symptoms, without more sophisticated or comprehensive analyses [3].

There are thus limited data on PROMs in patients recovering from COVID-19, despite this information being critical for optimising patient care. Our objective was to describe the impact of COVID-19 from the patient's perspective, including assessment of quality of life, frailty, dyspnoea, mood and sleep approximately 3 months after symptom onset.

A prospective consecutive cohort was recruited from the Post-COVID-19 Respiratory Clinic (PCRC) located in two academic hospitals. The PCRC was established to facilitate follow-up of all patients discharged after hospitalisation for COVID-19 in Vancouver, Canada. Referrals were received for patients as part of a standardised discharge protocol from all hospitals in the Vancouver Coastal Health Authority, which services over 1.25 million people in urban Vancouver and the coastal regions north of the city [4]. Patients aged 18 years or older who were hospitalised from March to June, 2020 with laboratory-confirmed severe acute respiratory syndrome coronavirus 2 (SARS-CoV-2) infection and able to complete surveys in English were included. There were no exclusion criteria. Informed consent was obtained from all patients (UBC Clinical Research Ethics Board \#H20-01239).

Patients completed questionnaires at the initial clinic visit approximately 3 months after initial symptom onset, including the 5-level EuroQoL 5-Dimensions (EQ-5D-5L), Frailty Index, University of California San Diego Shortness of Breath Questionnaire, Patient Health Questionnaire-9 and Pittsburgh Sleep Quality Index. We compared results to established age and population adjusted norms where available [5-8]. The provided normal threshold for dyspnoea is based on double the value of the minimum clinically important difference, given the absence of an established normal range [9]. Results were stratified by the presence or absence of baseline comorbidities as identified from the Charlson Comorbidity Index (CCI) [10]. The Frailty Index and CCI have previously been described and used to study patients with pulmonary conditions, such as interstitial lung disease [10-12]. A Wilcoxon rank sum test was used to determine whether there were differences in PROMs between patients who were and were not mechanically ventilated, using this variable as a marker of acute illness severity. All analyses were performed using $R$ version 3.6.3 (R Foundation).

A total of 101 patients were referred to the PCRC during the enrolment window, of whom 10 declined research participation, eight were seen virtually and could not complete questionnaires, and five did not meet criteria for laboratory-confirmed SARS-CoV-2. The final 78 included patients $(64 \%$ male) had a mean \pm SD age of $62 \pm 16$ years, and $32 \%$ were current or former smokers who had a median smoking

@ERSpublications

Over 75\% of patients admitted to hospital with COVID-19 have abnormal patient-reported outcome measures 3 months after symptom onset, with a third of patients reporting at least moderate impairment in major dimensions of quality of life https://bit.ly/32QMMgw

Cite this article as: Wong AW, Shah AS, Johnston JC, et al. Patient-reported outcome measures after COVID-19: a prospective cohort study. Eur Respir J 2020; 56: 2003276 [https://doi.org/10.1183/ 13993003.03276-2020]. 

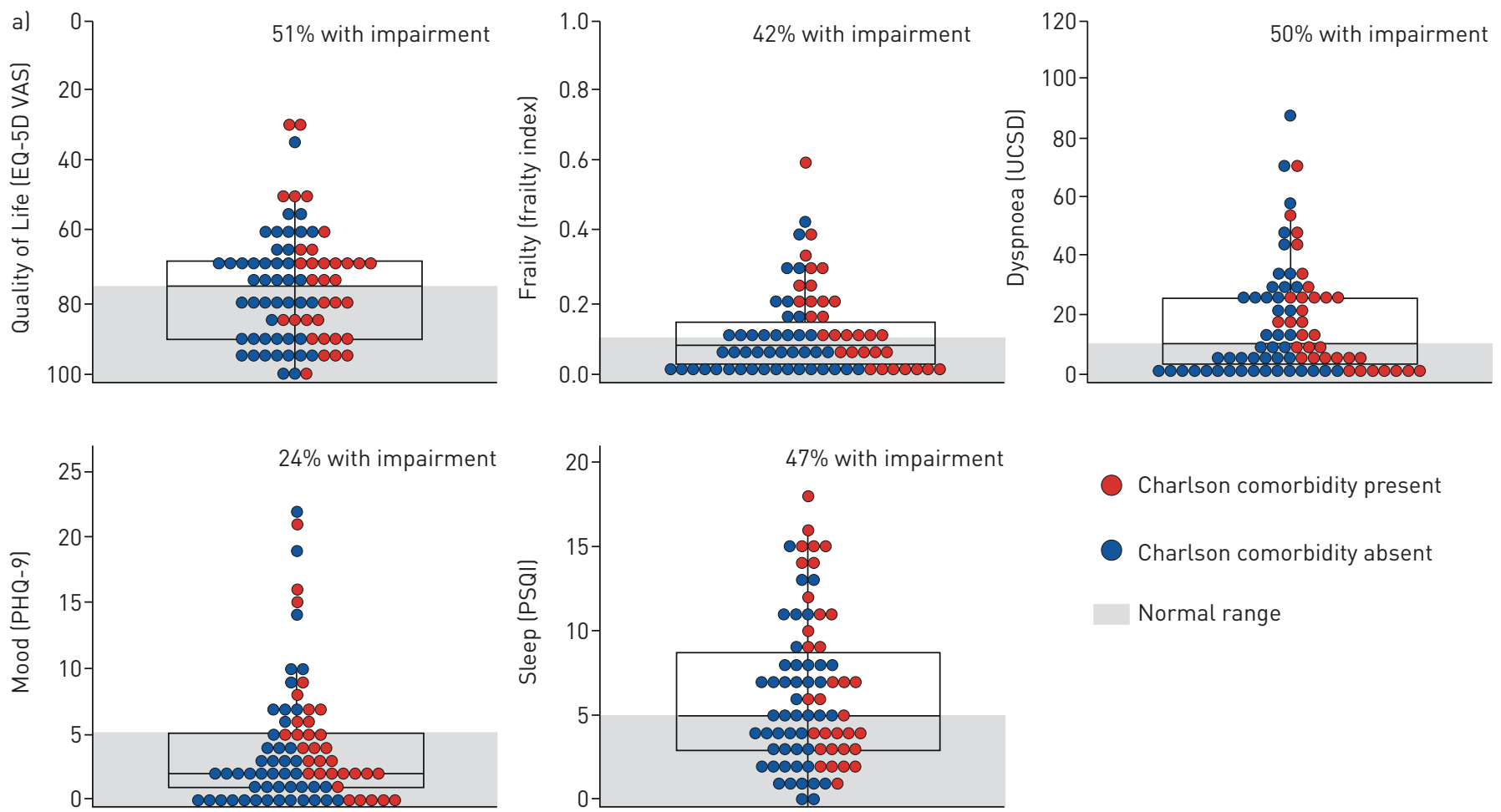

Charlson comorbidity present

Charlson comorbidity absent

Normal range

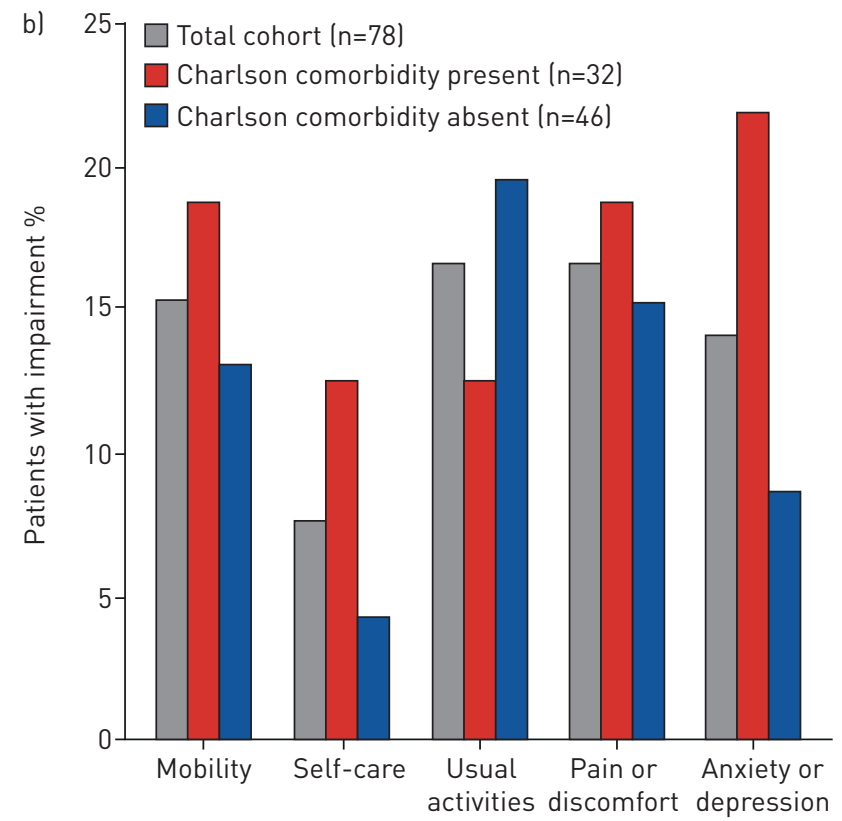

FIGURE 1 a) Patient reported outcomes 3 months after coronavirus disease 2019 (COVID-19) symptom onset stratified by the presence (red) or absence (blue) of Charlson comorbidities. Each circle represents a patient and the box represents the median and interquartile range. The y-axis shows the complete range of possible scores, and areas shaded in grey represent the normal range based on population adjusted norms where available. The provided normal threshold for dyspnoea is based on double the value of the minimum clinically important difference given the absence of an established normal range. b) Percentage of patients with impairment in each dimension of the 5-level EuroQoL 5-Dimensions (EQ-5D-5L). Results are shown for the total cohort (grey), and stratified by the presence (red) or absence (blue) of pre-existing Charlson comorbidities. Each dimension has five levels: no problems, slight problems, moderate problems, severe problems, and extreme problems. Patients who reported moderate problems or worse for a given dimension were considered to have impairment. PHQ: Patient Health Questionnaire; PSQI: Pittsburgh Sleep Quality Index; UCSD: University of California San Diego Shortness of Breath Questionnaire; VAS: Visual Analog Scale. 
history of 17 (interquartile range 1-31) pack-years. The most common pre-existing comorbidities identified by the CCI were diabetes $(26 \%)$, pulmonary disease $(8 \%)$, and history of myocardial infarction (8\%), with $59 \%$ of patients not having any comorbidities. Patients were assessed a median of 13 (interquartile range 11-14) weeks after symptom onset. At least one abnormal PROM was reported in $76 \%$ of patients, and 56\% had at least two abnormal PROMs (figure 1). The most frequently impaired outcomes were quality of life (51\%) and dyspnoea (50\%). $23 \%$ of patients reported the presence of cough. Of the patients with at least one Charlson comorbidity, $84 \%$ had an abnormal PROM and 38\% reported at least a moderate problem in one or more EQ-5D-5L dimensions. A higher proportion of these patients reported impairments with self-care and anxiety or depression compared to patients without any Charlson comorbidities at baseline ( $13 \%$ versus $4 \%$ for self-care, and $22 \%$ versus $9 \%$ for anxiety or depression). Of the patients without any Charlson comorbidities, 70\% had an abnormal PROM and 33\% reported at least a moderate problem in one or more EQ-5D-5L dimensions. A higher proportion of these patients reported impairment with usual activities compared to patients who had a Charlson comorbidity (20\% versus $13 \%$ ). There were no significant differences in PROMs between patients who were and were not mechanically ventilated.

In this prospective cohort, we show that $76 \%$ of patients admitted to hospital with COVID-19 have abnormal PROMs 3 months after symptom onset, with a third of patients reporting at least moderate impairment in major dimensions of quality of life. These findings were similar in patients with and without major pre-existing comorbidities, suggesting that many of these deficits are likely ongoing consequences of COVID-19. This adds further evidence that patients admitted to hospital with COVID-19 continue to struggle following recovery from the acute phase of this disease with a diverse range of impairments that extend beyond respiratory sequelae.

To our knowledge, this is the first study to report comprehensive PROMs in patients after hospitalisation for COVID-19. Our results are relevant to patients, clinicians and policymakers regarding long-term outcomes of COVID-19, supporting the design of appropriate discharge pathways, clinical standards and future studies that are aligned with patient needs. The importance of PROMs in understanding the impact of COVID-19 has been acknowledged with their inclusion in prospective registries [13]. A greater emphasis on integrating PROMs, in addition to increasing patient engagement, in future studies is needed to ensure that clinical and research efforts are truly patient-centred.

This study is limited by the lack of baseline PROMs; however, we categorised patients based on the presence or absence of Charlson comorbidities in an effort to address the impact that previous health status may have had on PROMs. Furthermore, we attempted to compare results to adjusted norms; however, such data were not available for all PROMs, which limits interpretation of the study findings. Age- and population-matched norms were available for EQ-5D-VAS, with more than half of previously hospitalised COVID-19 survivors having lower quality of life 3 months after symptom onset compared to other Canadians of similar age.

Over 27 million people have recovered from COVID-19 worldwide [14]; however, the long-term consequences of this disease remain largely unknown. Initial studies suggest that a significant proportion of patients are likely to have persistent physiological and radiographic abnormalities $[15,16]$. Our findings highlight the impact that COVID-19 has on patients even after reported recovery from acute manifestations of this disease, and emphasise the need for a comprehensive multidisciplinary approach to deliver the most appropriate care to these patients.

Alyson W. Wong ${ }^{1,2,5}$, Aditi S. Shah ${ }^{1,5}$, James C. Johnston ${ }^{1,3,4,6}$, Christopher Carlsten $\oplus^{1,3,6}$ and Christopher J. Ryerson ${ }^{1,2,6}$

${ }^{1}$ Dept of Medicine, University of British Columbia, Vancouver, BC, Canada. ${ }^{2}$ Centre for Heart Lung Innovation, University of British Columbia, Vancouver, BC, Canada. ${ }^{3}$ UBC School of Population and Public Health, Vancouver, BC, Canada. ${ }^{4} \mathrm{BC}$ Centre for Disease Control, Vancouver, BC, Canada. ${ }^{5}$ Contributed equally to this manuscript. ${ }^{6}$ Contributed equally to this manuscript.

Correspondence: Christopher J. Ryerson, St. Paul's Hospital, Ward 8B, 1081 Burrard St., Vancouver, Canada V6Z 1Y6. E-mail: chris.ryerson@hli.ubc.ca 1-604-806-8818

Received: 26 Aug 2020 | Accepted after revision: 16 Sept 2020

Conflict of interest: None declared.

Support statement: This study was supported by the TB Vets Charitable Foundation, Michael Smith Foundation for Health Research, Vancouver Coastal Health Research Institute and the University of British Columbia's Strategic Investment Fund. Funding information for this article has been deposited with the Crossref Funder Registry. 


\section{References}

1 Canadian Institute for Health Information. Patient-reported Outcome Measures (PROMs). www.cihi.ca/en/ patient-reported-outcome-measures-proms Date last accessed: 24 August 2020.

2 Zhou F, Yu T, Du R, et al. Clinical course and risk factors for mortality of adult inpatients with COVID-19 in Wuhan, China: a retrospective cohort study. Lancet 2020; 395: 1054-1062.

3 Carfi A, Bernabei R, Landi F, et al. Persistent symptoms in patients after acute COVID-19. JAMA 2020; 324: 603-605.

4 Vancouver Coastal Health. Who We Serve. www.vch.ca/about-us/who-we-serve Date last accessed: 24 Aug 2020.

5 Buysse D, Reynolds C III, Monk T, et al. The Pittsburgh Sleep Quality Index: a new instrument for psychiatric practice and research. Psychiatry Res 1988; 28: 193-213.

6 Janssen B, Szende A. Population Norms for the EQ-5D. In: Szende A, Janssen B, Cabases J, eds. Self-Reported Population Health: An International Perspective based on EQ-5D. Dordrecht, Springer, 2014; pp. 19-30.

$7 \quad$ Kroenke K, Spitzer RL, Williams JB. The PHQ-9: validity of a brief depression severity measure. J Gen Intern Med 2001; 16: 606-613

8 Rockwood K, Song X, Mitnitski A. Changes in relative fitness and frailty across the adult lifespan: evidence from the Canadian National Population Health Survey. CMAJ 2011; 183: E487-E494.

9 Eakin EG, Resnikoff PM, Prewitt LM, et al. Validation of a new dyspnea measure: the UCSD Shortness of Breath Questionnaire. University of California, San Diego. Chest 1998; 113: 619-624.

10 Charlson ME, Pompei P, Ales KL, et al. A new method of classifying prognostic comorbidity in longitudinal studies: development and validation. J Chronic Dis 1987; 40: 373-383.

11 Fisher JH, Kolb M, Algamdi M, et al. Baseline characteristics and comorbidities in the CAnadian REgistry for Pulmonary Fibrosis. BMC Pulm Med 2019; 19: 223.

12 Guler SA, Kwan JM, Leung JM, et al. Functional ageing in fibrotic interstitial lung disease: the impact of frailty on adverse health outcomes. Eur Respir J 2020; 55: 1900647.

13 Klok FA, Boon G, Barco S, et al. The Post-COVID-19 Functional Status scale: a tool to measure functional status over time after COVID-19. Eur Respir J 2020; 56: 2001494.

14 Worldometer. COVID-19 Coronavirus Pandemic 2020. www.worldometers.info/coronavirus/?zarsrc=130 Date last accessed: 9 October 2020

15 Liu C, Ye L, Xia R, et al. Chest CT and clinical follow-up of discharged patients with COVID-19 in Wenzhou City, Zhejiang, China. Ann Am Thorac Soc 2020; 17: 1231-1237.

16 Mo X, Jian W, Su Z, et al. Abnormal pulmonary function in COVID-19 patients at time of hospital discharge. Eur Respir J 2020; 55: 2001217. 